\title{
Notas sobre tres especies de Gigartinaceae (Rhodophyta) del litoral peruano
}

\author{
Notes on three species of Gigartinaceae (Rhodophyta) from Peruvian \\ coast
}

\author{
Martha Calderón', María Eliana Ramírez ${ }^{2}$ y Danilo Bustamante ${ }^{1}$
}

\begin{abstract}
1 Facultad de Ciencias Biológicas, Universidad Nacional Mayor de San Marcos. Ciudad Universitaria, Lima 1. Perú. Email Martha Calderón: marthacalderonrios@ gmail.com

2 Laboratorio Algas Marinas, Área Botánica, Museo Nacional de Historia Natural. Casilla 787 Santiago, Chile. Email María Eliana Ramírez: mramirez@mnhn.cl

Trabajo presentado a la XVIII Reunión Científica del Instituto de Investigaciones en Ciencias Biológicas Antonio Raimondi, "200 años del nacimiento de Charles Darwin y el 150 aniversario de la publicación de On the Origin of Species by Means of Natural Selection". Del 19 al 21 de agosto de 2009
\end{abstract}

Publicado impreso: 20/10/2010 Publicado online: $\quad 29 / 09 / 2010$

\section{Resumen}

La gran variabilidad morfológica de la familia Gigartinaceae ha producido constantes cambios taxonómicos en sus especies miembros. Tradicionalmente su taxonomía ha estado basada en caracteres de la morfología externa; sin embargo, recientes estudios morfológicos, respaldados con trabajos moleculares, han delimitado los géneros en base a al desarrollo del cistocarpo y de los tetrasporangios. El presente trabajo revisa y comenta sobre las estructuras morfológicas vegetativas y reproductivas de tres especies presentes en Perú: Chondracanthus chamissoi, Mazzaella denticulata y Chondrus canaliculatus.

Palabras clave: Género, morfología, reproductivo, distribución, taxonomía

\section{Abstract}

Gigartinaceae family has been under constant taxonomic changes due to the high morphologic variability of the species. Conventionally its taxonomy has been based in external morphology; nevertheless, recent morphologic studies, supported in molecular studies, have delimited the genus of this family on the basis of cystocarp development and the tetrasporangia. The vegetative and reproductive structures of three common species of the family present in the coast of Peru are described and commented: Chondracanthus chamissoi, Mazzaella denticulata and Chondrus canaliculatus.

Keywords: Genera, morphology, reproductive, distribution, taxonomy

\section{Introducción}

La Familia Gigartinaceae está caracterizada por: 1) Talo multiaxial con filamentos medulares y una corteza de pequeñas células. 2) El procarpo esta constituido por una célula de soporte, la rama carpogonial y un filamento lateral vegetativo. 3) La rama carpogonial posee 3 células que nacen de una célula de soporte la cual sirve de célula auxiliar. 4) Los filamentos gonimoblásticos se ramifican libremente a través de la medula y de filamentos secundarios, o penetran la envoltura del cistocarpo si está presente. 5) El tetrasporangio está dividido cruciadamente (Kylin 1932, Hommersand et al. 1993). Gigartinaceae incluye a siete géneros Sarcothalia, Gigartina, Iridaea, Rhodoglossum, Chondracanthus, Mazzaella y Chondrus.

La más reciente clasificación genérica de las Gigartinaceae sugerida por Hommersand et al. (1993), trajo consigo cambios taxonómicos para las especies presentes en el Perú.

Las especies del género Gigartina Stackhouse pasaron a formar parte del reestablecido género Chondracanthus Kützing por no diferenciarse una hilera externa e interna en la envoltura que rodea el cistocarpo, la formación de grupos de carposporangios a manera de racimos de uva y tetrasporangios formados en el límite entre la corteza y la médula (Hommersand et al. 1993, Hommersand et al. 1999).

Por otro lado, las especies pertenecientes al género Rhodoglossum pasaron al también al reestablecido género Mazzaella G. De Toni.

La presencia de una envoltura alrededor de la célula auxiliar, que luego es desplazada o reemplazada por los filamentos gonimoblásticos irregularmente ramificados, y la disposición única de los tetrasporangios en hileras derechas, formadas a partir de repetidas divisiones de células corticales superficiales hacia el interior del talo, constituyen los dos principales caracteres de diagnosis del género Rhodoglossum.
Mazzaella es distinguida por carecer de una envoltura alrededor del cistocarpo y por la presencia de filamentos tubulares que conectan los filamentos gonimoblásticos con células medulares y corticales internas.

Las especies pertenecientes a la familia Gigartinaceae presentes en el Perú son Chondracanthus chamissoi (C. Agardh) Kützing, Chondracanthus glomeratus (Howe) Guiry, Mazzaella hancockii (Dawson) Fredericq, Mazzaella denticulata (Dawson, Acleto et Foldvik) Fredericq y "Chondrus" canaliculatus (Homersand et al. 1993).

Con excepción de "Chondrus" canaliculatus (C. Agardh) Greville, las Gigartinaceae, en el Perú, han limitado sus caracterizaciones a descripciones del hábito y de la estructura vegetativa, sin considerar otros caracteres para su identificación, tales como el desarrollo morfológico de las estructuras reproductivas. Por tal motivo, el presente estudio se enfoca en tres especies, Chondracanthus chamissoi, Mazzaella denticulata y "Chondrus" canaliculatus, caracterizándolas morfológicamente y en función al desarrollo de las estructuras reproductivas.

\section{Materiales y métodos}

El material estudiado proviene de la Colección de Algas del Herbario del Museo Nacional de Historia Natural (SGO) de Santiago de Chile pertenecientes a las localidades de Nazca (bahía de San Juan) y Pisco (playa Mendieta). Además se realizaron colectas en la localidad de Lima (playas San Francisco, Ancón y playas de Barranco).

El análisis morfológico y morfoanatómico se realizó en material herborizado y en muestras conservadas en formalina/agua de mar 5\%. Se realizaron cortes microscópicos hechas a mano alzada, teñidas con azul de anilina $1 \%$ y montadas en Syrup Karo 30\%. Las estructuras vegetativas y reproductivas fueron observadas y fotografiadas bajo un microscopio. 


\section{Resultados}

\section{Chondracanhus chamissoi (C. Agardh) Kützing}

\section{Hábito}

El talo es de consistencia membranácea a cartilaginosa, presenta una coloración que va desde rojo purpúreo al verde oscuro, puede alcanzar los $36 \mathrm{~cm}$ de largo y hasta $1 \mathrm{~cm}$ de ancho (Fig. $1 \mathrm{y}$ 2). El talo esta constituido por un pequeño disco basal de fijación el cual puede llegar a medir $3 \mathrm{~mm}$ de diámetro, del cual emergen uno o varios estípites cilíndricos que van aplanándose hacia el ápice, el cual es agudo; puede ramificarse subdicotómicamente o no. En los márgenes laterales, presenta proliferaciones cuyo tamaño varían entre $0,1 \mathrm{~cm}$ y $11 \mathrm{~cm}$ y que también presentan pequeñas proliferaciones secundarias, variando en tamaño según la longitud de los diferentes ejemplares. Las proliferaciones presentan pequeñas pinnas dispuestas de manera alterna u opuesta. Los estípites de esta especie son variables en ancho, altura y grosor. Los talos pueden presentar márgenes dentados y pequeñas papilas en el centro del talo.

$\mathrm{Al}$ igual que los demás miembros de la familia, presenta un ciclo de vida isomórfico y trifásico, con alternancia de gametofitos y tetrasporofitos erectos. Los gametofitos son dioicos.

Estas plantas se distribuyen en el intermareal inferior y submareal superior, adheridas al sustrato rocoso, conchas de bivalvos y otros moluscos. Crece tanto en regiones expuestas al oleaje como protegidas. Esta especie es endémica de la costa del Pacifico Temperado Sudamericano, distribuyéndose desde Piura, en el norte del Perú, hasta Chiloé, al sur de Chile.

\section{Morfología vegetativa}

El talo es multiaxial y su espesor es de 319-326 $\mu \mathrm{m}$. La corteza esta constituida por 5 a 7 capas de células fuertemente pigmentadas de función fotosintética. Las primeras capas conformadas por células corticales superficiales de forma elipsoidal de 1,2-2,4 $\mu \mathrm{m}$ de ancho y 3,2 - 6,4 $\mu \mathrm{m}$ de largo. Las capas restantes están constituidas por células corticales internas pequeñas y redondas, de 3,2 - 6,4 $\mu \mathrm{m}$ de ancho y 3,6 - 4,4 $\mu \mathrm{m}$ de largo. Estas células están interconectadas con células vecinas y con células medulares a través de conexiones primarias y secundarias, lo que les confiere una forma más o menos estrellada. El grosor de la corteza varía entre $25,2-46 \mu \mathrm{m}$. La medula esta formada por filamentos medulares de 12,8 - 34,8 $\mu \mathrm{m}$ de largo y 3,2-6,4 $\mu \mathrm{m}$ de ancho, que se interconectan unas a otras por conexiones secundarias formando una especie de malla laxa (Fig. 3).

\section{Morfología reproductiva}

El gametófito femenino presenta las estructuras reproductivas principalmente dispuestas en las pinnas de las proliferaciones primarias y secundarias; los cistocarpos, esféricos y notorios, se distribuyen en los márgenes de dichas proliferaciones (Fig. 1). El procarpo se forma por modificación de una célula cortical interna para dar lugar a una célula de soporte que origina una rama carpogonial, compuesta por 3 células, y una rama estéril o filamento lateral vegetativo. La célula de soporte, que cumple la función de célula auxiliar, se fusiona con el carpogonio y recibe el núcleo diploide, modificando su forma y tamaño. No se observó la formación de protuberancias. La célula auxiliar es rodeada por filamentos medulares secundarios que formaran la envoltura interna que rodea el cistocarpo y que aparecen en estadios tempranos del desarrollo, inclusive antes de la aparición del primer filamento gonimoblásticos (Fig. 4). Más adelante la célula auxiliar da origen a los filamentos gonimoblásticos (Fig. 5). La envoltura interna, laxa y difusa, tiene un grosor aproximado de $8-15,6 \mu \mathrm{m}$, y esta compuesta por una hilera externa e interna. La hilera externa esta conformada por células grandes ricas en citoplasma, que se tiñen fuertemente, y están enlazadas por conexiones secundarias a células corticales internas y medulares. La hilera interna, a su vez, esta formada por células más delgadas y hialinas que se encuentran enlazadas directamente a los filamentos gonimoblásticos que dan origen a los grupos de carposporangios (Fig. 7). El cistocarpo maduro tiene forma ovoide y mide aproximadamente 150,4-181,6 $\mu \mathrm{m}$ de diámetro, esta constituido por abundantes carposporas de 1,6-6,4 $\mu \mathrm{m}$ de diámetro y por dos ostiolos bien definidos (Fig. 6).

En el tetrasporofito, al igual que el gametofito femenino, las estructuras reproductivas están confinadas a los márgenes de las proliferaciones, son de forma alargada y son ligeramente sobresalientes (Fig. 2). Los tetrasporangios están agrupados en la corteza formando un soro tetrasporangial de forma esférica, que mide entre 135,3 - 198,9 $\mu \mathrm{m}$ de diámetro (Fig. 8). Los tetrasporangios están divididos cruciada o decusadamente, y se forman a partir de células corticales internas (Fig. 9 y 10). Miden 43,5 - 63,2 $\mu \mathrm{m}$ de diámetro.

No se observaron ejemplares masculinos.

\section{Discusión}

Chondracanthus chamissoi tiene una morfología altamente variable. Algunos autores (Dawson et al. 1964) optaron por clasificar las distintas formas en dos grupos morfológicos, el grupo "lessonii" que corresponde a la forma de talo estrecho entre los 3-5 mm, y el grupo "chauvinii" referido a las formas de talo ancho, de $25 \mathrm{~mm}$ o más. Según lo mencionado, los especimenes estudiados en este trabajo corresponderían a una forma intermedia; sugiriendo más estudios para determinar su posición taxonómica.

El cistocarpo de Chondracanthus chamissoi es considerado como de tipo intermedio (Hommersand et al., 1999; Hommersand et al., 1993). En un cistocarpo de tipo primitivo (p.e. C. chapmanii de Nueva Zelanda), las células no modificadas de la envoltura presentan el núcleo haploide pequeño y el diploide alargado. Un cistocarpo de tipo avanzado (p.e. C. teedei de Europa) tiene la célula auxiliar con un núcleo largo, niveles amplificados de DNA y células de la envoltura alargadas conteniendo numerosos núcleos haploides. Sin embargo en este trabajo no se ha podido determinar al cistocarpo de Chondracanthus chamissoi como característico del tipo intermedio.

Por otro lado, el cistocarpo de los especimenes estudiados, está más o menos compartimentalizado, siendo morfológicamente intermedio entre Chondracanthus, donde los carposporangios forman un denso tejido (Hommersand et al. 1993, fig. 22- 24; Hughey \& Hommersand 2008, fig. 9- 16) y Gigartina, que es completamente compartimentalizado (Hommersand et al. 1999, fig. 27).

Según lo observado, la envoltura que rodea al cistocarpo no es completamente compacta, los largos filamentos medulares secundarios que la conforman se disponen de manera laxa y 


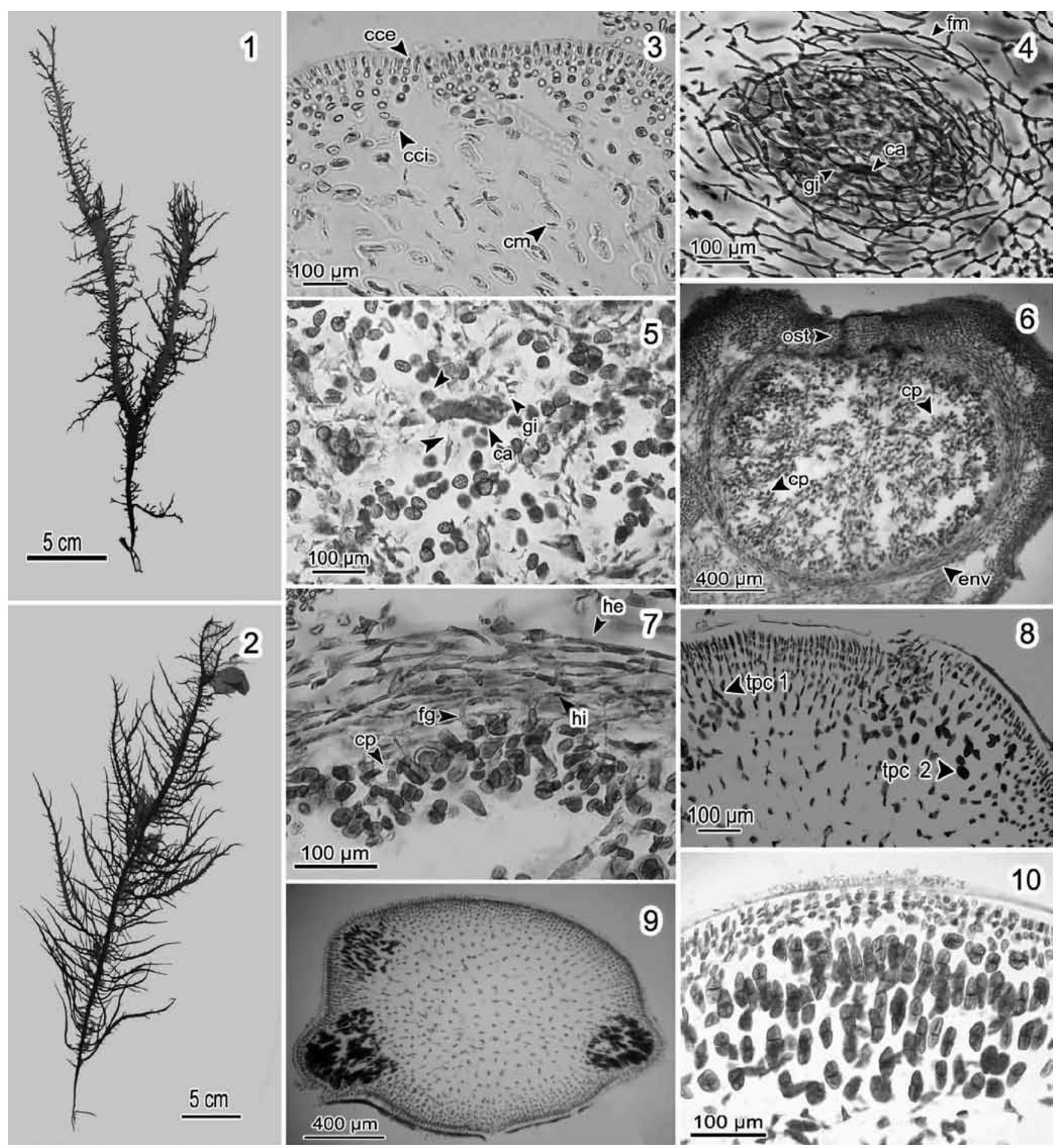

Figuras 1-10. Chondracanthus chamissoi. (1) Hábito del gametofito femenino. (2) Planta tetrasporofítica. (3) Estructura vegetativa del talo mostrando las células corticales externas (cce), células corticales internas (cci) y células medulares (cm). (4 y 5) Estadio temprano de desarrollo de la célula auxiliar (ca) después de la fertilización originando filamentos gonimoblásticos iniciales (gi). Nótese que se encuentra rodeada por filamentos medulares primarios ( $\mathrm{fm}$ ). (6) Cistocarpo maduro rodeado de una envoltura (env), mostrando dos ostiolos (ost) y carposporas (cp) agrupadas. (7) Filamentos gonimoblasticos (fg) que originan grupos de carposporas (cp) y se enlazan a las células de la hilera interna (hi) de la envoltura; he: células de la hilera externa de la envoltura. (8) Formación de tetrasporocitos a partir de filamentos primarios de células corticales internas; trp1 y trp2: tetrasporocitos en diferentes estadios de desarrollo. (9 y 10) Soro tetrasporangial ubicado en la corteza interna.

difusa, no correspondiendo a lo descrito por Hommersand et al. (1993), para el género Chondracanthus, sin embargo los caracteres restantes caen dentro de dicha descripción.

El género Gigartina difiere principalmente de Chondracanthus, en que la envoltura no esta diferenciada en una hilera externa e interna, la formación de grupos de carposporangios a manera de racimos de uva, los tetrasporangios se forman en el limite entre la corteza y la medula, generalmente a partir de filamentos secundarios, y las estructuras reproductivas femeninas están formadas sobre ramas secundarias, mientras que en Chondracanthus, se forman sobre ramas ordinarias (Hommersand et al. 1993; Hommersand et al. 1999).

Finalmente, se puede concluir en base al material analizado, que Chondracanthus chamissoi presenta una envoltura laxa y difusa que rodea el cistocarpo, dicha envoltura se puede diferenciar en dos hileras según el tamaño y contenido de los filamentos que 
la integran, y el cistocarpo es más o menos compartimentalizado, ya que los carposporangios forman pequeños grupos separados por filamentos gonimoblásticos alargados y hialinos. Según lo analizado en el presente estudio, Chondracanthus chamissoi exhibe caracteres intermedios entre los géneros Chondracanthus y Gigartina, lo que motivará el desarrollo de futuros estudios, morfológicos y moleculares, que develen la posición taxonómica de esta especie.

\section{Mazzaella denticulata (Dawson, Acleto et Foldvik) Fredericq}

\section{Hábito}

Talo solitario, de coloración rojo vinoso purpúreo a negruzco; presenta un pequeño disco basal de fijación menor de $1 \mathrm{~mm}$ de diámetro, del cual deriva erguida una estípite cilíndrica corta de $1-2 \mathrm{~mm}$ de largo, continuado en una lámina foliácea en forma de cinta de $15,9-47,8 \mathrm{~cm}$ de largo y $0,8-2,9 \mathrm{~cm}$ de ancho (Fig. 11 y 12). Presenta ramificación irregular, dicotómicamente o no, con proliferación y ramas marginales en los bordes que llegan a medir 13,4 cm de largo y $8 \mathrm{~mm}$ de ancho. El margen es entero, la superficie lisa y la consistencia cartilaginosa.

Esta planta se encuentra localizada en la zona de intermareal inferior y submareal superior, en zonas expuestas fuertemente al oleaje, lo que dificulta su recolección. Se adhieren al sustrato rocoso. En ocasiones pueden encontrarse algunos ejemplares varados en las playas arenosas. Esta especie es endémica a la costa del Pacifico Temperado Sudamericano. En la costa peruana solo se han colectado ejemplares en las playas de Barranco, Lima, localidad tipo de esta especie. En la costa chilena, esta especie ha sido reportada, en la Isla Alacrán, en la región de Arica. Por lo tanto esta especie se distribuiría desde Lima, en el centro del Perú, hasta Arica en el norte de Chile.

\section{Morfología vegetativa}

El talo es multiaxial, cuyo espesor es de 87,6 - 103,2 $\mu \mathrm{m}$. Esta constituido por una corteza de 23,6 - 28,6 $\mu \mathrm{m}$ de grosor, con $4-7$ capas de células. La corteza externa la conforman 4 - 5 capas de células isodiamétricas de 1,6 - 3,0 $\mu \mathrm{m}$ de largo y 1,6-2,4 $\mu \mathrm{m}$ de ancho, que van aumentando de tamaño hacia el interior del talo y adoptando una forma estrellada, por las conexiones primarias y secundarias que tiene con células vecinas. Estas células conforman la corteza interna con $2-3$ capas de células, de 1,6 - 2,4 $\mu \mathrm{m}$ de largo y 3,2 - 3,4 $\mu \mathrm{m}$ de ancho. La médula esta conformada por largas y pocas células filamentosas de 21,6 - 31,2 $\mu \mathrm{m}$ de largo y 0,6 - 1,0 $\mu \mathrm{m}$ de ancho, enlazadas unas con otras por conexiones secundarias, constituyendo así una médula laxa y suelta (Fig. 13).

\section{Morfología reproductiva}

Las estructuras reproductivas femeninas se encuentran distribuidas en toda la superficie de la lámina sobresaliendo notoriamente de la misma (Fig. 11). El procarpo se forma a partir de divisiones seriadas de una célula cortical interna, el cual da lugar a la célula de soporte, la célula inicial de la rama carpogonial y la célula inicial del filamento estéril. La célula de soporte es la de mayor tamaño, más basal y la más teñida. La rama carpogonial esta formada por 3 células, siendo el carpogonio la célula apical que origina un tricógeno largo y de contenido hialino (Fig. 14). La célula de soporte, que cumple la función de célula auxiliar, se fusiona con el carpogonio y recibe el núcleo diploide, modificando su forma y tamaño observándose pequeñas protuberancias que darán origen a los gonimoblastos iniciales (Fig. 15). A medida que se va desarrollando el cistocarpo, las células gonimoblásticas dan origen a cadenas de carposporangios de dos a tres células de largo. El cistocarpo no presenta una envoltura que la rodea (Fig. 17); sin embargo se observa que los filamentos gonimoblásticos, que son altamente ramificados, se enlazan a las células corticales y medulares internas por medio de filamentos tubulares delgados, desde estadios tempranos del desarrollo del cistocarpo (Fig. 16 y 18). Los cistocarpos maduros, de 149,2 189,6 $\mu \mathrm{m}$ de diámetro, tienen forma elíptica y se encuentran inmersos en el talo. Están constituidos de abundantes carposporas de $1,6-4,8 \mu \mathrm{m}$ de diámetro.

En el tetrasporofito, al igual que el gametofito femenino, las estructuras reproductivas están dispersas en la superficie de la lámina, sobresaliendo ligeramente (Fig. 12). Los tetrasporangios están agrupados en la medula formando un soro tetrasporangial, de forma elíptica que mide entre 94,6 - 134,8 $\mu \mathrm{m}$ de diámetro, y que se ubica en la medula (Fig. 20). Los tetrasporangios están divididos cruciada o decusadamente, formándose a partir de filamentos corticales internos secundarios; además, se encuentran enlazados a otras células por conexiones secundarias (Fig. 19). Los tetrasporangios miden $2,2-7,8 \mu \mathrm{m}$ de diámetro.

No se observaron ejemplares masculinos.

\section{Discusión}

El cistocarpo carece de envoltura; sin embargo, en algunas ocasiones se presentan células medulares primarias que la rodean parcialmente y que se forman en los últimos estadios del desarrollo del carposporofito. Por otro lado, los filamentos gonimoblásticos de la periferia, se unen a estas células medulares primarias y a células corticales internas, que cumplen una función nutritiva, a través de filamentos largos hialinos de poco contenido citoplasmático, algunos delgados y otros tubulares. Generalmente, los filamentos gonimoblásticos se enlazan a las células corticales internas por medio de numerosos filamentos delgados, mientras que cuando se enlazan a células medulares lo hacer por medio de los filamentos tubulares.

Mazzaella denticulata, al igual que $M$. californica, especie holotipo, no presenta filamentos gametofititos secundarios; a diferencia de $M$. splendens que si los presenta, sin embargo coincide con este ultimo en el tamaño del cistocarpo, siendo pequeño en $M$. californica. Por otro lado, $M$. denticulata forma los tetrasporangios sólo a partir de filamentos corticales internos secundarios, a diferencia de $M$. affinis que los desarrolla enteramente de filamentos corticales primarios, o de $M$. splendens que los forma de filamentos primarios y secundarios. Esto concuerda parcialmente con lo referido por Hommersand et al. (1999), quienes mencionan que las especies de Sudamérica y Sudáfrica poseen el cistocarpo con escasos filamentos secundarios, y los tetrasporangios son formados enteramente de filamentos secundarios.

Los caracteres antes mencionados para $M$. denticulata, como la falta de una envoltura alrededor del cistocarpo, filamentos gonimoblásticos muy ramificados, límite distinguible entre gonimoblastos y células vegetativas, y la presencia de cadenas carpogoniales originadas de células gonimoblásticas, concuerdan con los caracteres de diagnosis del género Mazzaella; sin em- 


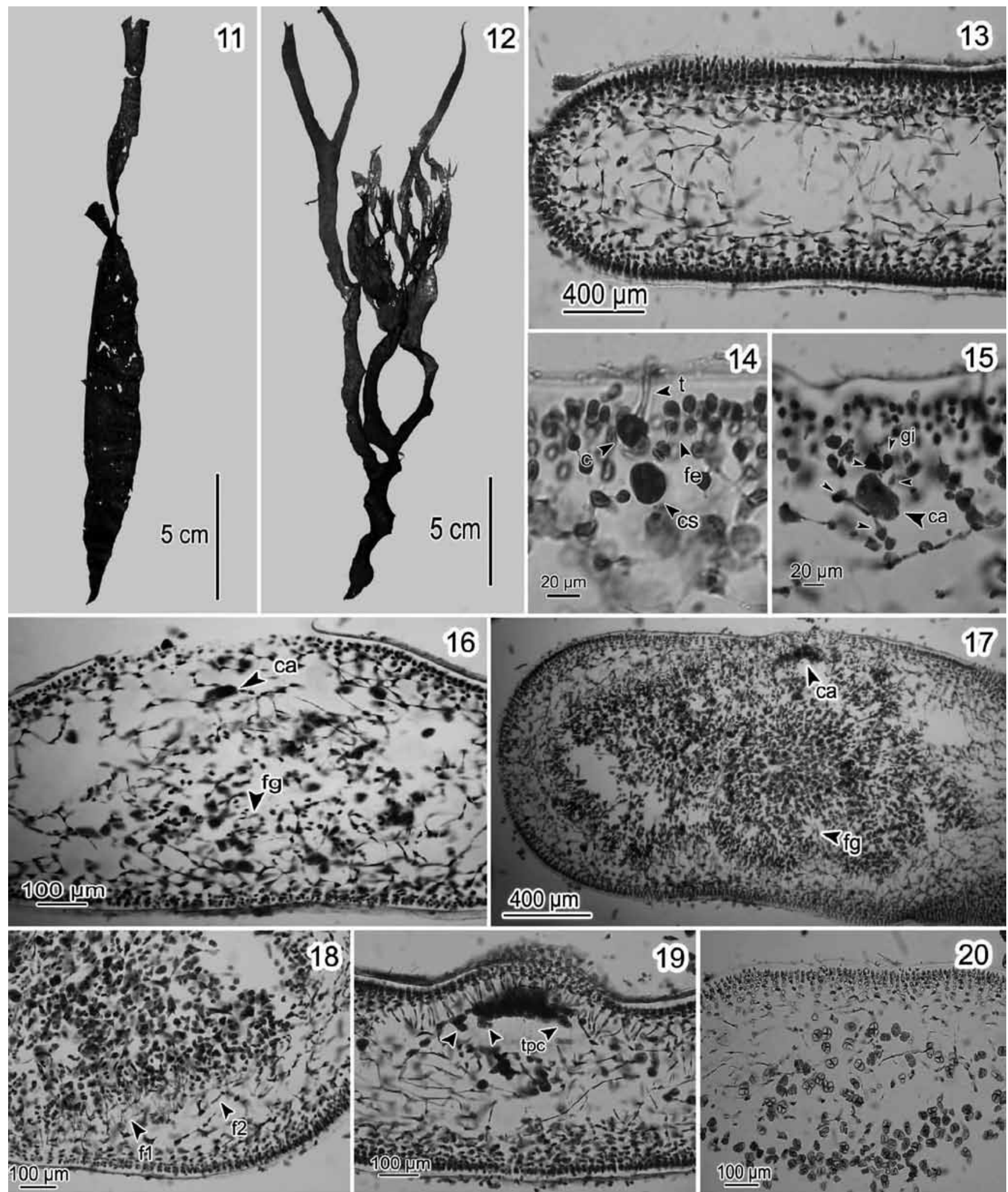

Figuras 11 - 20. Mazzaella denticulata. (11) Hábito del gametofito femenino. (12) Planta tetrasporofítica. (13) Estructura vegetativa del talo mostrando la corteza y la medula filamentosa. (14) Procarpo formado por una célula de soporte (cs), un filamento estéril (fe) y la rama carpogonial que contiene al carpogonio (c) que origina al tricógino (t). (15) Célula auxiliar (ca) que origina numerosas protuberancias que darán lugar a los gonimoblastos iniciales (gi). (16) Cistocarpo en desarrollo mostrando célula auxiliar (ca) y filamentos gonimoblásticos (fg). (17) Cistocarpo maduro con ausencia de una envoltura. (18) Filamentos que unen los filamentos gonimoblásticos a células de la corteza interna (f1) y a células medulares (f2). (19) Tetrasporocitos (tpc) formados a partir de los filamentos corticales secundarios internos. (20) Soro tetrasporangial ubicado en la región medular. 
bargo, a diferencia de otras especies, posee pocas protuberancias que originan gonimoblastos iniciales y carecen de filamentos secundarios o filamentos gametofititos secundarios.

Este es el segundo reporte en el Perú desde su descripción (como Rhodoglossum denticulatum) y es el primero como Mazzaella denticulata.

\section{“Chondrus” canaliculatus (C. Agardh) Grevile}

\section{Hábito}

Talo membranáceo y cartilaginoso, de color verde oscuro a rojo purpúreo; presenta un pequeño disco de fijación de $1-2$ $\mathrm{mm}$ de diámetro, que origina un pequeño estípite erecto y cilíndrico, de 1,6 - 2,4 mm de largo, que origina una fronda aplanada (Fig. 21 y 22). El talo mide $11,2-24,1 \mathrm{~cm}$ de longitud y $0,5-1,3 \mathrm{~cm}$ de ancho. Presenta ramificación subdicotómica de hasta 10 órdenes, en algunas ocasiones sin patrón de ramificación definido. Las ramas miden entre 5,8 - 10,8 cm de largo y $0,6-1,1 \mathrm{~cm}$ de ancho y presentan pequeńas proliferaciones en los márgenes enteros de las ramas. La morfología de esta especie es variable en largo, ancho y grosor.

Esta especie se encuentra ubicada en la zona intermareal inferior y submareal superior, de zonas protegidas y poco expuestas al oleaje. Se adhieren fuertemente al sustrato rocoso y valvas de moluscos. Esta especie es endémica a la costa del Pacifico Temperado Sudamericano, distribuyéndose desde las islas Chincha, en la costa central del Perú, hasta Chiloé, en el sur de Chile.

\section{Morfología vegetativa}

El talo es de construcción multiaxial, cuyo espesor es de 169,6 - 190,4 $\mu \mathrm{m}$; conformado por una corteza de 13,2 - 15,2 $\mu \mathrm{m}$ de grosor, con 5 a 6 capas de células. Las células corticales más externas, de forma alargada, de 1,2 - 2,2 $\mu \mathrm{m}$ de largo y $0,4-0,8 \mu \mathrm{m}$ de ancho, forman de 4 a 5 capas. Las células corticales internas, de 1,0 - 1,6 $\mu \mathrm{m}$ de largo y 2,0 - 3,2 $\mu \mathrm{m}$ de ancho, con 1 a 2 capas; adquieren una forma estrellada debido a las conexiones secundarias con células vecinas. La médula es filamentosa y laxa, conformada por células de 10,8 - 24,4 $\mu \mathrm{m}$ de largo y 0,6- 0,8 $\mu \mathrm{m}$ de ancho (Fig. 23).

\section{Morfología reproductiva}

Las estructuras reproductivas femeninas son globosas y sobresalientes, se encuentran distribuidas en la cara externa superficie de la fronda del gametofito (Fig. 21). El procarpo lo conforman una célula de soporte, que más adelante actúa de célula auxiliar, un filamento estéril, y la rama carpogonial de tres células (Fig. 24). Una vez ocurrida la fertilización, el carpogonio se fusiona con la célula auxiliar, que modifica su forma y tamaño para dar paso a la formación de muchas protuberancias que originaran los gonimoblastos iniciales que crecen y forman los filamentos gonimoblásticos (Fig. 25). La célula auxiliar esta rodeada por una envoltura más o menos compacta, de 15,2 - 25,2 $\mu$ m organizada a partir de filamentos secundarios originados de las células medulares vegetativas del gametofito (Fig. 26). Los filamentos gonimoblásticos no penetran la envoltura ni se unen a las células que la conforman. Las células gonimoblásticas originan cadenas de carposporangios. Los cistocarpos maduros, de 117,2 - 183,2 $\mu \mathrm{m}$ de diámetro, son elípticos y están inmersos en la médula. Las carposporas miden 1,6-2,8 $\mu \mathrm{m}$ de diámetro.
En el tetrasporofito las estructuras reproductivas, también protuberantes y sobresalientes, se encuentran en las ramas cerca de los ápice (Fig. 22). Los tetrasporangios están agrupados en soros elípticos de 126,6 $\mu \mathrm{m}$ de diámetro (Fig. 28). Los tetrasporangios, de 3,2 - 5,2 $\mu \mathrm{m}$ de diámetro, se forman a partir de filamentos medulares secundarios, en la medula externa y están divididos cruciada y decusadamente (Fig. 27).

No se observaron ejemplares masculinos.

\section{Discusión}

Esta especie fue tratada y estudiada ampliamente por Arakaki et al. (1997), mencionando que "Chondrus" canaliculatus de Chile y Perú no corresponden con los caracteres de diagnosis para el género Chondrus, basados sobre la especie holotipo Chondrus crispus Stackhouse, ni a otros géneros de la familia. Hommersand et al. (1999), refieren que "Ch". canaliculatus morfológicamente es intermedio entre Mazzaella y el clado Iridea/Sarchotalia de Chile, Nueva Zelanda y Sudáfrica, ya que los filamentos de la envoltura desarrollan gradualmente y son más difusos que en Iridea o Sarcothalia, además que el tetrasporangio se forma en filamentos secundarios en la médula externa, como en Sarcothalia. La presencia de una envoltura alrededor del cistocarpo y el origen de los tetrasporangios a partir de filamentos secundarios de la medula externa son los dos principales caracteres en conflicto que sugieren que "Ch". canaliculatus debe ser removida del género Chondrus y constituir un nuevo género dentro de la familia Gigartinaceae.

\section{Agradecimientos}

Al Consejo Nacional de Ciencia, Tecnología e Innovación Tecnológica (CONCYTEC) de Perú y al Programa de Movilidad Estudiantil del Vicerrectorado Académico de la Universidad Nacional Mayor de San Marcos, por el financiamiento de viajes y manutención. Al Museo Nacional de Historia Natural de Santiago Chile por las facilidades otorgadas para la realización de este trabajo. Un especial agradecimiento a la Prof. Olga Riofrio por el incentivo y sugerencias.

\section{Literatura citada}

Arakaki N., M.E. Ramírez \& C. Córdova. 1997. Desarrollo morfológico y taxonomía de Chondrus canaliculatus (C. Ag.) Greville (Rhodophyta, Gigartinaceae) de Perú y Chile. Bol. Mus. Nac. Hist. Nat. Chile 46:7-22

Dawson E.Y., C. Acleto \& N. Foldvik. 1964. The seaweeds of Perú. Beihefte Nova Hedwigia 13: 1-111, 81 plates.

Hommersand M.H. \& S.fredericq. 1990. Sexual reproduction and cystocarp development. In Cole\& Sheath (eds.). Biology of the red algae. New York, Cambridge University Press. P 305-345

Hommersand M.H., M. Guiry, S. Fredericq \& G. Leisler. 1993. New perspectives in the taxonomy of the Gigartinaceae (Gigartinales, Rhodophyta). Hidrobiologia 260/261: 105-120

Hommersand M.H., S. Fredericq \& W. Freshwater. 1994. Phylogenetics systematics and biogeography of the Gigartinaceae (Gigartinales, Rhodophyta) based on sequence analysis of rbcL. Botanica Marina. 37: 193-203.

Hommersand M.H., S. Fredericq, D.W. Freshwater \& J. Hughey. 1999. Recent development in the systematics of the Gigartinaceae (Gigartinales, Rhodophyta) based on rbcL sequences analysis and morphological evidence. Phyclogical Research 47: 139-151 

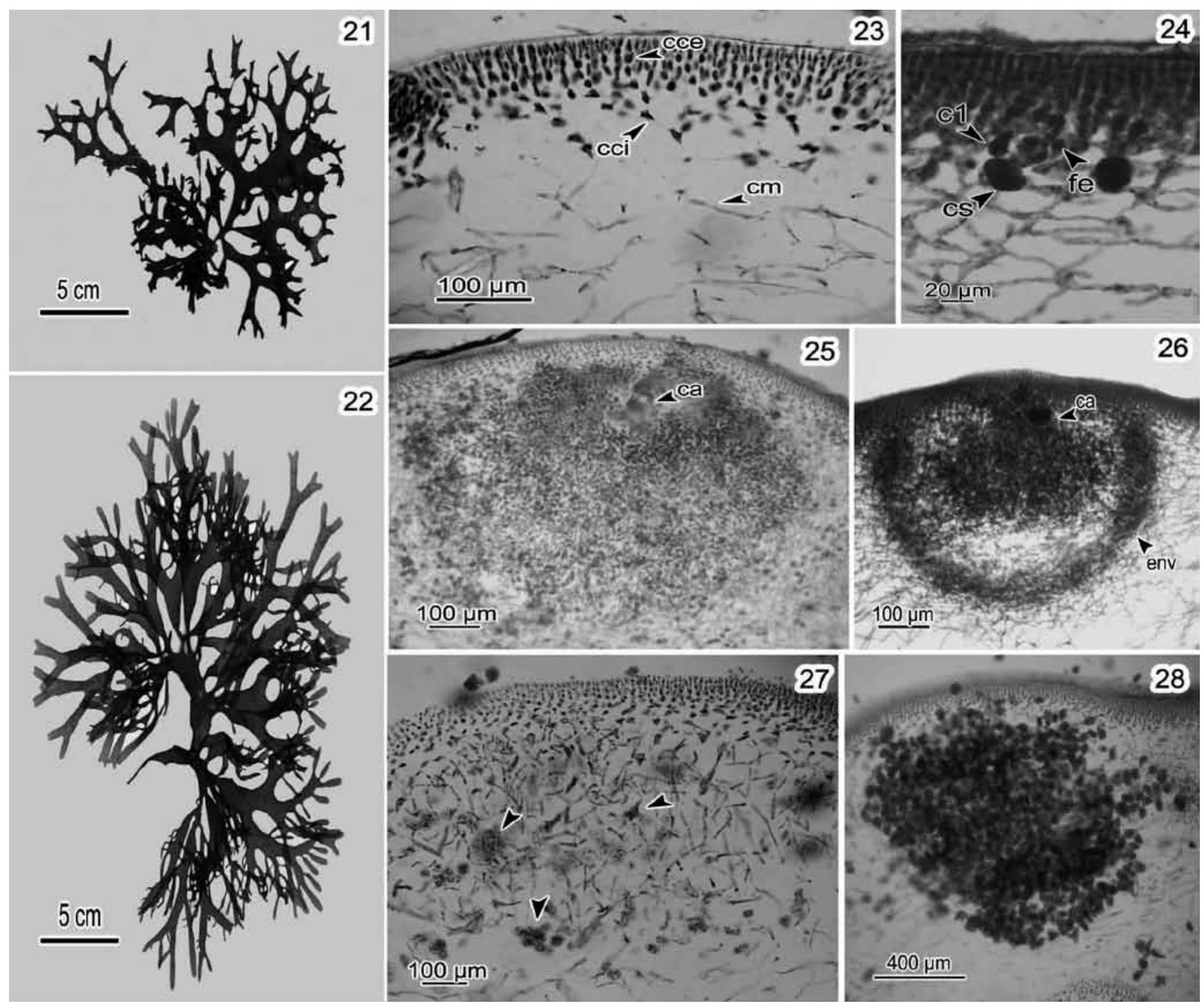

Figuras 21 - 28. Chondrus canaliculatus. (21) Hábito del gametofito femenino (Ica: Pisco, leg. César Acleto 17- VIII- 1978, SGO 136498). (22) Planta tetrasporofítica (Ica: Pisco, leg. César Acleto 17- VIII- 1978, SGO 136500). (23) Estructura vegetativa del talo mostrando las células corticales externas (cce), células corticales internas (cci) y células medulares $(\mathrm{cm})$. (24) Procarpo formado por una célula de soporte (cs), un filamento estéril (fe) y una celula inicial (c1) que dará origen a la rama carpogonial. (25) Cistocarpo en formación mostrando la célula auxiliar (ca). (26) Cistocarpo maduro rodeado de una envoltura (env). (27) Tetrasporocitos (cabeza de flechas) formados a partir de filamentos medulares secundarios. (28) Soro tetrasporangial ubicado en la médula.

Hughey J.R. \& M.H. Hommersand. 2008. Morphological and molecular systematics stuffy of Chondracanthus (Gigartinaceae, Rhodophyta) from Pacific North America. Phycologia 47 (2): 124-155.
Kylin H. 1932. Studien ubre die Entwicklunschichte der Florideen. K. Sv. Vet. Akad. ANLD. 63 (11): 1' 139, 82 figs. 


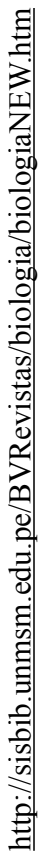

